\title{
Spectral Energy Distributions of 3C 279 revisited: BeppoSAX observations and variability models
}

\author{
L. Ballo ${ }^{1}$, L. Maraschi ${ }^{1}$, F. Tavecchio ${ }^{1}$, A. Celotti ${ }^{2}$, G. Fossati ${ }^{3}$, G. Ghisellini ${ }^{4}$, E. Pian $^{5}$,

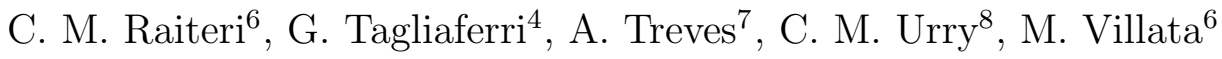

Received —

\footnotetext{
${ }^{1}$ Osservatorio Astronomico di Brera, via Brera 28, 20121 Milano, Italy

${ }^{2}$ Scuola Internazionale Superiore di Studi Avanzati/International School for Advanced Studies, Astrophysics Sector, via Beirut 4, 34014 Trieste, Italy

${ }^{3}$ Center for Astrophysics and Space Sciences, University of California, San Diego, 9500 Gilman Drive, Code 0424, La Jolla, CA 92093, USA

${ }^{4}$ Osservatorio Astronomico di Brera, via Bianchi 46, 23807 Merate, Italy

${ }^{5}$ Osservatorio Astronomico di Trieste, via G. B. Tiepolo 11, 34131 Trieste, Italy

${ }^{6}$ Osservatorio Astronomico di Torino, Strada Osservatorio 20, 10025 Pino Torinese (TO), Italy

${ }^{7}$ Dipartimento di Fisica, Università dell'Insubria, via Valleggio 11, 22100 Como, Italy

${ }^{8}$ Yale Center for Astronomy and Astrophysics, Yale University, New Haven, CT 208121, USA
} 


\begin{abstract}
We present the BeppoSAX observations of 3C 279 performed in 1997 January 13 - 23 simultaneously with $\gamma$-ray pointings with EGRET on board CGRO and optical observations at the Torino Observatory (1997 January 11 - 18). ISO data close to this epoch are also available. We compare the derived Spectral Energy Distribution with those obtained at all other epochs with adequate multiwavelength coverage. Simple spectral models fitted to the multiepoch SEDs suggest that changes in intensity can be ascribed mainly to the variation of the bulk Lorentz factor of the plasma in the jet.
\end{abstract}

Subject headings: quasars: general — quasars: individual (3C 279) - X-rays: spectra — radiation mechanisms: non-thermal 


\section{Introduction}

In the last ten years about 60 blazars (BL Lac objects and flat-spectrum radio quasars) have been detected in $\gamma$-rays by EGRET (Mukherjee et al. 1997, Hartman et al. 1999). The observations show that a large fraction of the total power is emitted in this band, and many of these objects exhibit rapid variability (on timescales of hours or days) at the highest energies. The high $\gamma$-ray luminosities and short variability timescales impose strong and general constraints on the emitting region; in particular, independent of other evidence, the $\gamma$-ray transparency condition implies that the emitting plasma moves relativistically.

The mechanism of $\gamma$-ray production is most likely inverse Compton scattering: the seed photons could be produced both within the jet via synchrotron radiation, which is responsible for the emission from radio to UV, (Synchrotron Self Compton [SSC], see Maraschi, Ghisellini \& Celotti 1992), and outside the jet (External Compton [EC]) by the accretion disk (Dermer \& Schlickeiser 1993) or by the broad line region clouds (Sikora, Begelman \& Rees 1994). The relative weight of the two sources of seed photons is given by the ratio of their respective energy densities as seen in the frame of the emitting matter (Sikora, Begelman \& Rees 1994). In flat spectrum radio quasars, since the external photons typically have much higher frequencies than synchrotron photons, when both processes SSC and EC are important the former dominates the SED at low energies (in the X-ray band, see Kubo et al. 1998), the latter in the $\gamma$-ray range; significant information about the internal structure of the jet can thus be obtained from observations covering the full X-ray to $\gamma$-ray spectral region.

Another open problem is the physical cause of the variability. Flares could be due to propagating shocks causing transient acceleration of relativistic electrons (see e.g. Marscher \& Gear 1985). Recently an idea initially proposed by Rees (1978) was worked out and applied in particular to 3C 279 by Spada et al. (2001)(see also Ghisellini 1999): 
briefly, the collision of shock fronts travelling in the jet with different bulk Lorentz factors can yield efficient impulsive particle acceleration leading to enhanced radiation (Spada et al. 2001; Ghisellini 1999).

In any case both the synchrotron and inverse Compton emission from a single electron population are expected to vary in a correlated fashion, but for a given change in the electron spectrum the amplitudes of the SSC and EC variations will be different (Ghisellini \& Maraschi 1996, Sikora 1997). Furthermore inhomogeneities (clouds) in the medium close to the jet may act as mirrors for the synchrotron photons (Ghisellini \& Madau 1996, Boettcher \& Dermer 1998, Bednarek 1998). Variability may also result from changes in the beaming factor, due to geometric effects (see Villata \& Raiteri 1999). All of these hypotheses predict a particular relative variability in the synchrotron and inverse Compton components, so a study of simultaneous variations in different bands can provide important information about the nature of phenomena occurring in blazar jets.

3C $279(z=0.538)$ was the first blazar discovered as a $\gamma$-ray source with the Compton Gamma Ray Observatory (CGRO; Hartman et al. 1992) and one of the brightest in this band, and as such was the target of several multifrequency campaigns (for the most recent re-analysis of all the data see Hartman et al. 2001, hereafter H01). It was chosen for observations with BeppoSAX (1997 January 13 - 23) simultaneous with an EGRET pointing and optical observations (1997 January 11 - 18) to improve our knowledge of the detailed spectral shape from X-rays to $\gamma$-rays and to continue the study of its variability at high energies. Since BeppoSAX covers a very wide energy range $(0.1-100 \mathrm{keV})$, it is ideal to study the connection between the $\mathrm{X}$ and $\gamma$-ray continuum where the transition from the SSC to the EC process should take place. In the present work a complete analysis of the BeppoSAX observations and associated SED are fully presented, combined with a 
discussion of the historical SEDs of 3C 279 with best multiwavelength coverage. Preliminary results were published in Maraschi et al. (1998), Maraschi (2000), Maraschi et al. (2000), Maraschi \& Tavecchio (2001a) and Maraschi \& Tavecchio (2001b). The BeppoSAX data are also included in $\mathrm{H} 01$.

The structure of the paper is the following: in $\S 2$ we present the analysis and results of the BeppoSAX observations of 1997; in $\S 3$ we construct the overall SED of 1997 and compare it with other emission states for which simultaneous or quasi-simultaneous multifrequency data are available; in $\S 4$ we apply a simple spectral model including both the SSC and EC processes to reproduce the observed SEDs at different epochs and derive physical parameters for the emitting plasma. Conclusions are presented in $\S 5$. Throughout the paper we assume $H_{0}=65 \mathrm{~km} \mathrm{~s}^{-1} \mathrm{Mpc}^{-1}$ and $q_{0}=0.5$.

\section{BeppoSAX observations of 1997}

The scientific payload of the Italian-Dutch X-ray satellite BeppoSAX円 (see Boella et al. 1997) consists of four coaligned Narrow Field Instruments (NFIs) and two Wide Field Cameras. Two of the NFIs use concentrators to focalize X-rays: the Low Energy Concentrator Spectrometer (LECS; one unit) has a detector sensitive to soft-medium X-ray photons (0.1-10 keV), while the Medium Concentrator Spectrometer (MECS; three units) detects photons in the energy range 1.3-10 keV. The Phoswich Detector System (PDS), sensitive from 15 to $300 \mathrm{keV}$ nominally, consisting of four identical units, uses rocking collimators so as to monitor source and background simultaneously with interchangeable units. We will not consider here data from the fourth NFI, a High Pressure Gas Scintillation Proportional Counter (HPGSPC).

\footnotetext{
1 http://www.sdc.asi.it
} 
The observations of 3C 279 were planned to be simultaneous with a continuous pointing at the source with EGRET and with optical observations performed at the Torino Observatory. They consisted of 5 pointings, distributed over 10 days (1997 January 13 - 23) to cover the typical two-week duration of an EGRET observation. Exposure times and observed count rates in the various detectors are reported in the BeppoSAX journal of observations (see Table [1). We searched for variability both within each observation and between the different observations using the $\chi^{2}$ test against constancy: no significant flux variations were detected; we therefore combined all the observations deriving a cumulative spectrum.

The BeppoSAX spectral data were analyzed using the standard software packages XSELECT (v1.4) and XSPEC (v10.0) and the September 97 version of the calibration files released by the BeppoSAX Scientific Data Center (SDC). From the event files we extracted the LECS and MECS spectra in circular regions centered around the source with radii of $8^{\prime}$ and $6^{\prime}$ respectively (see the SAX Analysis Cookbook (2). The PDS spectra extracted with the standard pipeline with the rise-time correction were provided directly by the BeppoSAX SDC.

For the spectral analysis we considered the LECS data in the restricted energy range 0.1-4 keV, because of unsolved calibration problems at higher energies. PDS data above $180 \mathrm{keV}$ were discarded since the count rate was too close to the instrumental detection limit to be reliable. Background spectra extracted from blank field observations at the same detector position as the source were used for background subtraction in the LECS and MECS, while for the PDS the simultaneously measured off-source background was used. We fitted rebinned LECS, MECS and PDS net spectra jointly, allowing for two normalization factors to take into account uncertainties in the intercalibration of different

2 ftp://www.sdc.asi.it/pub/sax/doc/software_docs/saxabc_v1.2.ps.gz 
instruments (see SAX Cookbook).

The total LECS+MECS+PDS spectrum is well described by a single power-law model, with an absorption column consistent with the galactic value $\left(N_{H}=(2.21 \pm 0.1) \cdot 10^{20} \mathrm{~cm}^{-2}\right.$, as reported in Elvis, Wilkes \& Lockman 1989); the results of the spectral fits are summarized in Table 2. The residuals of the fit show a weak excess around $E \simeq 0.5 \mathrm{keV}$ in the LECS data (see Fig. [1], upper panel): we modelled the whole spectrum with either a broken power law or a power law with a black body component. In both cases the excess observed at low energy is partly accounted for (see Fig. [1], lower panel), but requires a column

density higher than the galactic value. In any case the reduction in $\chi^{2}$ is not significant and we considered only the power law model in the rest of our analysis.

All through the analysis the LECS/MECS normalization ratio was allowed to vary; the best fit value obtained is 0.85 , in agreement with the intervals proposed by SAX Cookbook. We fixed the intercalibration between PDS and MECS to the usual value, 0.85: the fit with a free normalization factor gives a slightly higher value (even if in the acceptable range), without considerable improvement in the $\chi^{2}$.

\section{Spectral Energy Distributions and models}

\subsection{7}

Simultaneously to the BeppoSAX observations 3C 279 was monitored in $\gamma$-rays by EGRET and in the optical at the Torino Observatory. The $\gamma$-ray data are reported in H01.

R, V and B band magnitudes were obtained between 1997 January 11 - 18 at the 1.05 meter REOSC telescope, equipped with a $1296 \times 1152$ pixels Astromed CCD camera with a 0.467 arcsec/pixel image scale. Data were reduced with the ROBIN procedure locally developped, which includes bias subtraction, flat field correction, and circular Gaussian 
fit. Magnitudes are reported in Table 3. A search in the UMRAO database provided radio data at $4.8 \mathrm{GHz}$ (1997 January 21 - 22), 8.0 GHz (1997 January 24) and 14.5 GHz (1997 January 14).

In 1996 December 19 (only one month before the BeppoSAX pointings), 3C 279 was observed by ISOPHOT, the photometer on board ISO, as part of a program involving numerous AGN. The source was detected in seven bands, from $4.8 \mu \mathrm{m}$ to $170 \mu \mathrm{m}$; results are reported in Haas et al. (1998).

All the above fluxes together with the deconvolved BeppoSAX $0.1-100 \mathrm{keV}$ spectrum are shown in Fig. [2]. The X-ray spectrum is flat, while the connection with the EGRET $\gamma$-ray data indicates a steepening of the SED between the two ranges. The $\gamma$-ray flux is slightly higher than that measured in 1993, when the source was in a very low emission state (see the next section); the same occurs for the optical luminosity.

Finally, it is notable that the far infrared peak observed by ISO dominates the bolometric luminosity. Integrating the measured fluxes, Haas et al. (1998) find $L_{I R}=2 \cdot 10^{47} \mathrm{erg} \mathrm{s}^{-1}$. This value is similar to that found for three other flat spectrum radio quasars examined by the same authors; however, while in the other FSRQs the infrared emission is a smooth continuation of the synchrotron spectrum, in the case of $3 \mathrm{C} 279$ the IR spectrum shows a narrow peak suggestive of a thermal origin.

However previous observations with IRAS in four bands from $12 \mu \mathrm{m}$ to $100 \mu \mathrm{m}$ (see Impey \& Neugebauer 1988, Moshir 1990) yielded a $60 \mu \mathrm{m}$ flux density and three upper limits lower than the fluxes measured by ISO, principally at $60 \mu \mathrm{m}$ (a factor of $\sim 9$ ). If this discrepancy is real (see Haas et al. 1998) the implied variability would not be consistent with thermal emission from a region of about $1 \mathrm{kpc}$.

Moreover from the shape of the ultraviolet spectrum in the lowest state one can derive 
an upper limit of $\sim 2 \cdot 10^{45} \mathrm{erg} \mathrm{s}^{-1}$ to the UV luminosity of a possible accretion disk in 3C 279 (Pian et al. 1999). Since in the case of 3C 279 the line of sight is at small angle to the axis of the relativistic jet which is presumably close to that of the putative accretion disk, it is difficult to suppose the existence of a hidden nuclear UV component with intensity similar to that of the IR emission. Thus it is not easy to understand how the dust could be heated unless the AGN underwent a long phase of higher activity in the past or a highly luminous starburst is taking place in this galaxy.

\subsection{Multiepoch SEDs}

One of the characteristic properties of blazars is the extreme variability, observed at all wavelengths but much accentuated at high energies. Understanding this trait can provide important information about mechanisms operating in the inner regions of the source. Therefore, after collecting data provided from the campaign organized in 1997, we revisited several SEDs of 3C 279 obtained with simultaneous or quasi-simultaneous multiwavelength data, with the scope of comparing different states of emission. In the following we briefly recapitulate basic information on the SEDs used: the first refers to the epoch of the $\gamma$-ray discovery of 3C 279 in 1991, the other three are based on organized campaigns with wide multiwavelength coverage (1993 and 1996 pre-flare, flare).

1991: The first detection of $\gamma$-ray emission dates from 1991 June 16 - 28: the flux measured by EGRET was very intense, with evidence of a flare on a timescale of days (Kniffen et al. 1993); a second observation (1991 October 3 - 17) revealed a lower flux level. A literature search provided quasi-simultaneous data to the EGRET observations (see Hartman et al. 1996; Bonnell, Vestrand \& Stacy 1994) to assemble the SED shown in Fig. [3] (upper panel). IR data were obtained the 1991 June 16 and the optical one is an average over the epoch of the $\gamma$-ray flare, while the UV 
observations date from 1991 July 27, without resorting to the correlation between UV and R-band fluxes applied in Hartman et al. (1996).

1993: This first multiwavelength campaign organized after the discovery found $3 \mathrm{C} 279$ in a low state: the emission had faded dramatically at all frequencies above $10^{14} \mathrm{~Hz}$, while the flux variations at radio to millimeter wavelengths were minor. To assemble the SED in Fig. [3] (lower panel) we used data from Maraschi et al. (1994), but differently from that work the EGRET observation (having a $4 \sigma$ level) is taken as an upper limit.

1996: Throughout this second multiwavelength campaign 3C 279 was in a high state, similar to that of 1991; during the observation there was an extraordinary flare lasting 2-3 days, particularly intense in the $\gamma$-ray band. Following Wehrle et al. (1998), we divided the light curve in two parts, averaging the observed fluxes in the period January $24-28$, preceding the flare, and in a 2-day window centered on the $\gamma$-ray peak. The resulting SEDs are shown in Fig. [4].

\section{Interpretation}

In order to reproduce the observed SEDs we used a model with minimal assumptions. The emitting region is described as a homogeneous sphere with radius $R$ endowed with a magnetic field $B$ and moving with bulk Lorentz factor $\Gamma$; the line of sight is at an angle $\theta$ with respect to the jet axis, so that the Doppler factor $\delta=1 /[\Gamma(1-\beta \cos \theta)]$ is relevant for relating observed quantities with rest frame quantities. The source is filled with relativistic electrons, whose energy spectrum is described by a broken power law between $\gamma_{\min }$ and $\gamma_{\max }$, which is determined by four parameters, the break energy $\gamma_{b}(\gg 1)$, the normalization $k$ and the spectral indices of the asymptotic power laws below and above the break, 
respectively $n_{1}<3$ and $n_{2}>3$ (see Tavecchio, Maraschi \& Ghisellini 1998):

$$
N(\gamma)=k \gamma^{-n_{1}}\left(1+\frac{\gamma}{\gamma_{b}}\right)^{n_{1}-n_{2}}
$$

This form for the distribution function has been assumed in order to describe the curved shape of the SED. We used $\gamma_{\max }=5 \cdot 10^{4}$ for all the states. The value of the minimum energy of the scattering electrons is uncertain. We assumed $\gamma_{\min }=1$ as inferred in the case of other emission line blazars from the lack of spectral breaks in the soft X-ray continuum (see Tavecchio et al. 2000).

Electrons emit via the synchrotron and inverse Compton mechanisms. At low frequencies the synchrotron spectra are limited by the self-absorption frequency. Below that frequency the model has the standard self-absorbed spectrum with slope $5 / 2$. Additional emission components from regions farther out in the jet are necessary to account for the spectra at lower frequencies, as indeed expected if the flat radio spectra of blazars are due to the superposition of different self-absorbed components from different locations in the jet. The SEDs calculated here refer to the innermost emitting region.

The inverse Compton spectra have been calculated using the full Klein-Nishina cross-section (Jones 1968; see also Blumenthal \& Gould 1970) and taking into account the beaming of the external radiation in the blob frame as pointed out in Dermer (1995). There the author uses a single power law as electron distribution and a monochromatic external radiation. In our case the flux calculation can be made only numerically because we use a more complex electron distribution.

We neglected the direct contribution of photons from the accretion disk since it is strongly red-shifted in the comoving frame when the bulk Lorentz factor is large and the $\gamma$-ray emission occurs at distance $\geq 10^{17} \mathrm{~cm}$ (Sikora, Begelman \& Rees 1994). The dominant contribution to the external radiation can be identified with that produced by the BLR via reflection/reprocessing of the disk emission. The spectrum of the BLR 
is described by a black body peaking at a frequency $\nu_{e x t}=10^{15} \mathrm{~Hz}$ with a luminosity $L_{b l r}$ diluted in a spherical region with radius $R_{b l r}$. For 3C 279 the BLR luminosity was estimated in Celotti, Padovani \& Ghisellini (1997) from the observed emission lines (see also Francis et al. 1991) as $L_{b l r}=6 \cdot 10^{44} \mathrm{erg} \mathrm{s}^{-1}$. This value corresponds to $\sim 30 \%$ of the accretion disk luminosity estimated by Pian et al. (1999) from the UV observations during the 1993 campaign. Applying the model to the 1993 state, the one with lowest emission, we found that the best reproduction was obtained for an energy density $u_{b l r} \sim 10^{-4} \mathrm{erg} \mathrm{cm}^{-3}$; this corresponds to a BLR radius $R_{b l r}=4 \cdot 10^{18} \mathrm{~cm}$, consistent with the value obtained from the $R_{b l r}-L_{b l r}$ relation found by Kaspi et al. (2000). This value was then kept fixed for all states.

We also estimated the possible contribution of the observed IR radiation. From the luminosity and temperature given by Haas et al. (1998) we derived the minimum radius of the emitting region, $R_{\min }=\sqrt{\frac{L_{I R}}{4 \pi \sigma T_{I R}^{4}}} \sim 10^{20}-10^{21} \mathrm{~cm}$, and a maximum energy density $u_{I R} \sim 10^{-8}-10^{-5} \mathrm{erg} \mathrm{cm}^{-3}$, negligible with respect to the BLR energy density calculated above.

Finally, modelling the emission from blazars it is common to adopt the relation $\delta \sim \Gamma$, corresponding to an angle of view $\theta=1 / \Gamma$. However studying different states of the same object we intended to allow variations of $\Gamma$ and $\delta$ but not of the angle of sight; so we fixed it to a value $\theta=3^{\circ}$, leaving the Lorentz factor $\Gamma$ free to vary and computing the corresponding value of $\delta$.

The computed models are shown together with the data in Figs. [2], [3] and [4] with the different components (synchrotron, disk radiation, SSC and EC) as well as their sum plotted separately. In Fig. [5] the models (sum of all the contributions) reproducing the SEDs of the different states are compared with each other.

We tried to reproduce the different levels varying the smallest possible number of 
parameters. Our models are consistent with the observed variations being essentially due to the bulk Lorentz factor $\Gamma$ (also in the short timescale flare observed in 1996 ); the parameters required are given in Table $₫$. The goodness of the choice of a $\gamma_{\min }=1$ was verified a posteriori: considering all the states we found that if $\gamma_{\min }>5$ the models cannot reproduce the hard $\mathrm{X}$-ray/soft $\gamma$-ray data. The magnetic field is $\simeq 0.5 \mathrm{G}$ in all states, while the size of emitting region and the Doppler factor used allow variability timescales $t_{v a r}=\frac{R}{\delta c}$ from 1 to 2 days, consistent with the observations. We note that the small angle of view $\left(\theta=3^{\circ}\right)$ and the high bulk Lorentz factor (from 6 to 17) used involve relatively high superluminal speeds, from $\beta_{a p p} \sim 3-4$ in the low states to $\beta_{a p p} \sim 12-15$ in the high levels. Superluminal velocities observed in 3C 279 range from $3 c-5 c$ (Unwin et. al. 1989; Carrara et al. 1993) to $4.8 c-7.5 c$ derived from a long-term high-frequency VLBI monitoring of six superluminal components in the relativistic jet in 3C 279 (see Wehrle et al. 2001). Higher velocities have also been measured in the past: Cotton et. al. (1979) originally found a value of $15 c$ (speeds have been expressed assuming $H_{0}=70 \mathrm{~km} \mathrm{~s}^{-1} \mathrm{Mpc}^{-1}$ and $q_{0}=0.1$ ). These velocities refer however to regions further away along the jet, while our data concern the emission from much smaller scales. Thus the comparison, though interesting, can only be indicative. We would like to mention that in the internal shock model it is predicted that shells with high $\Gamma$ will slow down to an average value of $\Gamma$ further out.

In the models above the X-ray emission is attributed to the SSC mechanism, while the $\gamma$-ray emission is ascribed to the EC process; in this case, supposing a variation in the bulk Lorentz factor $\Gamma$, one expects that the relative change of the peak emission of these two components follows the relation $F_{E C} \propto F_{S S C}^{3 / 2}$ (see Ghisellini \& Maraschi 1996). We searched for a correlation between X-ray and $\gamma$-ray fluxes in 3C 279 using all the available simultaneous measurements in the two bands, derived from H01; in Fig. [6] we plot $\nu_{\gamma} F_{\gamma}$ vs $\nu_{X} F_{X}$. The X-ray data are not completely homogeneous. When possible, we used the flux measured at $3 \mathrm{keV}$. Otherwise we took the available flux at a slightly 
different energy $(\sim 2 \mathrm{keV})$. For the EGRET data $\nu_{\gamma}$ is taken at $167 \mathrm{MeV}$ (frequency at which observations for all the states are given in H01). In some cases H01 gives two fluxes corresponding to energies of $121 \mathrm{MeV}$ and $208 \mathrm{MeV}$ respectively. In these cases we used an average flux. For all the measurements the plotted errors are the maximum between the value provided and the $5 \%$ of the flux, in order to take into account intercalibration problems. The straight line, obtained with a fit that considered the errors in both ranges, has slope $1.49 \pm 0.13$, in good agreement with the expectation.

\section{Discussion and Conclusions}

We have analyzed the X-ray spectra of 3C 279 obtained from the BeppoSAX satellite in 1997 January. The result of our analysis is that the featureless X-ray continuum in the $2-100 \mathrm{keV}$ energy band is well represented by a power-law with $\alpha \sim 0.66$. The observations from BeppoSAX were part of an organized campaign involving also $\gamma$-ray and optical measurements; adding radio data from the UMRAO database and IR data from ISO, 1 month earlier than the high energy observations, a quasi-simultaneous SED from radio to $\gamma$-rays was derived.

We also revisited the overall SEDs of four other different states of 3C 279 with wide simultaneous spectral coverage, from 1991 to 1997. We modelled the observed SEDs with

the widely used homogeneous synchrotron-Inverse Compton model, estimating the physical parameters in the emission region. This was done using the minimum possible number of free parameters.

The model used in this work (simpler than the one adopted in H01) is fully specified by eight parameters: the four parameters of the electron distribution, the magnetic field, the size of the emitting region, the bulk Lorentz factor and the angle of view. In addition 
the external radiation energy density and the typical frequency of the photons enter in the model. The latter is practically fixed at $\nu_{\text {ext }} \simeq 10^{15} \mathrm{~Hz}$, while the former has been inferred estimating the luminosity and the dimension of the BLR: so they can't be considered free parameters. From an observed SED we can in principle obtain six quantities, namely the synchrotron peak frequency and luminosity, the inverse Compton peak frequency and luminosity and the spectral indices of the synchrotron component after the peak and that of the inverse Compton component before the peak (connected to the indices of the electron distribution $n_{2}$ and $n_{1}$ respectively). In addition to these six quantities the typical variability timescale can give an upper limit to the size of the source. Fixing the angle of view, the total number of observational constraints is equal to the number of parameters of the model which can therefore in principle be fully constrained (see Tavecchio et al. 2000). In the case of 3C 279 the main observational uncertainty is the position of synchrotron peak, falling in the poorly covered FIR band. However given the fact that we consider 5 states, we feel that within the model assumptions our parameter determination is robust.

We confirm that the bulk of the $\gamma$-ray $(\mathrm{MeV}-\mathrm{GeV})$ emission can be modelled as IC radiation produced by electrons in the jet upscattering soft ambient photons reprocessed in the Broad Line Region as originally proposed by Sikora, Begelman \& Rees (1994) (for a recent review of blazar models, see Sikora \& Madejski 2001) while the X-ray continuum should be due to Synchrotron-Self Compton radiation. Notably the relation between the $\gamma$-ray and X-ray fluxes of all the available simultaneous observations follows the relation expected under this hypothesis.

The most interesting result of our study is that the SEDs of the 5 different states considered here can be reproduced varying in a substantial way only one parameter, namely the bulk Lorentz factor $\Gamma$ of the emitting region, and considering all the other physical quantities (size, magnetic field, electron distribution) as almost constant. A significant 
variation of $\Gamma$ can be understood if the jet is energized by the injection of shells with initially different values of the bulk Lorentz factor as proposed in the internal shock model (see Spada et al. 2001; Ghisellini 1999). The collision between successive shells naturally predicts the production of a new shell with an intermediate $\Gamma$ on short timescales as necessary in the case of the 1996 flare.

Other causes of variability may coexist with the above as it is well possible to reproduce the observed variability varying more than one parameter. Even then however, it is not possible to reproduce the variability behaviour without varying $\Gamma$ (H01). In particular we cannot exclude that the peaks of the main emission components shift to higher energies in brighter states. This is in fact suggested by the hardening of the $\gamma$-ray spectrum. However the position of the synchrotron peak in this source as well as in many emission line blazars is difficult to constrain since it falls in the FIR band where observations are difficult.

Even taking into account the above uncertainties it seems that the variability behaviour of 3C 279 differs form that of Mkn 501, one of the best studied and most "extreme" or "high energy peaked" BL Lac objects in the spectral sequence discussed by Fossati et al. (1998) (see Costamante et al. 2001). In the latter case the large spectral variability observed in X-rays on short and long timescales, causing a substantial shift in the peak of the synchrotron emission, appears to be mainly due to the variation of the energy of electrons emitting at the peak (those at $\gamma_{b}$, Tavecchio et al. 2001) presumably due to a change in the acceleration process not associated with a significant change in $\Gamma$. In fact the emission of Mkn 501 in the soft X-ray band varies much less than in the medium-hard band implying strong intrinsic spectral changes and small if any variations in $\Gamma$, at least within a homogeneous model.

These issues are clearly important for a physical understanding of the variability of blazars which could be driven by somewhat different processes in different "types" 
of blazars (emission line blazars vs. BL Lacs or high luminosity vs. low luminosity, see Ghisellini et al. 1998, Ghisellini \& Celotti 2001). Simultaneous access to X-ray, FIR and $\gamma$-ray plus ground based facilities as will be possible in the next years will allow us to test new ideas in this field. 
We thank the BeppoSAX SDC for providings us with the cleaned data. This research has made use of data from the University of Michigan Radio Astronomy Observatory which is supported by funds from the University of Michigan. We used also the NASA/IPAC Extragalactic Database (NED), which is operated by the Jet Propulsion Laboratory, California Institute of Technology, under contract with the National Aeronautics and Space Administration. This work was partly supported by the Italian Ministry for University and Research (MURST) under grant Cofin2000 and by the Italian Space Agency (ASI) under grant I-R-105-00. 


\section{REFERENCES}

Bednarek, W. 1998, A\&A, 336, 123

Blumenthal, G. R. \& Gould, R. J. 1970, Reviews of Modern Physics, 42, 237

Boella, G., Butler, R. C., Perola, G. C., Piro, L., Scarsi, L., \& Bleeker, J. A. M. 1997, A\&AS, 122, 299

Boettcher, M. \& Dermer, C. D. 1998, ApJ, 501, L51

Bonnell, J. T., Vestrand, W. T., \& Stacy, J. G. 1994, ApJ, 420, 545

Carrara, E. A., Abraham, Z., Unwin, S. C., \& Zensus, J. A. 1993, A\&A, 279, 83

Celotti, A., Padovani, P., \& Ghisellini, G. 1997, MNRAS, 286, 415

Costamante, L. et al. 2001, A\&A, 371, 512

Cotton, W. D. et al. 1979, ApJ, 229, L115

Dermer, C. D. \& Schlickeiser, R. 1993, ApJ, 416, 458

Dermer, C. D. 1995, ApJ, 446, L63

Elvis, M., Wilkes, B. J., \& Lockman, F. J. 1989, AJ, 97, 777

Fossati, G., Maraschi, L., Celotti, A., Comastri, A., \& Ghisellini, G. 1998, MNRAS, 299, 433

Francis, P. J., Hewett, P. C., Foltz, C. B., Chaffee, F. H., Weymann, R. J., \& Morris, S. L. 1991, ApJ, 373, 465

Ghisellini, G. \& Madau, P. 1996, MNRAS, 280, 67 
Ghisellini, G. \& Maraschi, L. 1996, Blazars Continuum Variability, ASP Conference Series, Vol. 110, Miller, H. R., Webb, J. R. \& Noble, J. C. eds.

Ghisellini, G., Celotti, A., Fossati, G., Maraschi, L., \& Comastri, A. 1998, MNRAS, 301, 451

Ghisellini, G. 1999, Astronomische Nachrichten, 320, 232

Ghisellini, G., \& Celotti, A. 2001, Invited talk at "Issues of unifications of AGNs", (Marciana Marina, May 2001), R. Maiolino, A. Marconi \& N. Nagar, (astro-ph/0108110)

Haas, M., Chini, R., Meisenheimer, K., Stickel, M., Lemke, D., Klaas, U., \& Kreysa, E. 1998, ApJ, 503, L109

Hartman, R. C. et al. 1992, ApJ, 385, L1

Hartman, R. C. et al. 1996, ApJ, 461, 698

Hartman, R. C. et al. 1999, ApJS, 123, 79

Hartman, R. C. et al. 2001, ApJ, 553, 683

Impey, C. D. \& Neugebauer, G. 1988, AJ, 95, 307

Jones, F. C. 1968, Phys. Rev., 167, 1159

Kaspi, S., Smith, P. S., Netzer, H., Maoz, D., Jannuzi, B. T., \& Giveon, U. 2000, ApJ, 533, 631

Kniffen, D. A. et al. 1993, ApJ, 411, 133

Kubo, H., Takahashi, T., Madejski, G., Tashiro, M., Makino, F., Inoue, S., \& Takahara, F. 1998, ApJ, 504, 693

Maraschi, L., Ghisellini, G., \& Celotti, A. 1992, ApJ, 397, L5 
Maraschi, L. et al. 1994, ApJ, 435, L91

Maraschi, L. et al. 1998, The Active X-ray Sky: Results from BeppoSAX and RXTE, 453, L. Scarsi, H. Bradt, P. Giommi \& F. Fiore eds.

Maraschi, L. 2000, Proceedings of the 20th Texas Symposium (Austin, December 2000; astro-ph/0107565

Maraschi, L., Chiappetti, L., Fossati, G., Pian, E., \& Tavecchio, F. 2000, Advances in Space Research, Vol. 25, 713

Maraschi, L., \& Tavecchio, F. 2001a, Blazar Demographics and Physics, ASP Conf. Series, P. Padovani \& C. M. Urry eds., in press (astro-ph/0102295)

Maraschi, L., \& Tavecchio, F. 2001b, Proceedings of "X-ray astronomy 2000" (Palermo, September 2000), R. Giacconi, L. Stella \& S. Serio eds., ASP Conf. Series, in press (astro-ph/0107566)

Marscher, A. P. \& Gear, W. K. 1985, ApJ, 298, 114

Moshir, M. E. A. 1990, IRAS Faint Source Catalogue, version 2.0 (1990), 0

Mukherjee, R. et al. 1997, ApJ, 490, 116

Pian, E. et al. 1999, ApJ, 521, 112

Rees, M. J. 1978, MNRAS, 184, 61P

Sikora, M., Begelman, M. C., \& Rees, M. J. 1994, ApJ, 421, 153

Sikora, M. 1997, AIP Conf. Proc. 410: Proceedings of the Fourth Compton Symposium, 494

Sikora, M., \& Madejski, G. 2001, Invited talk at "International Symposium on High Energy Gamma-Ray Astronomy", Heidelberg, F. Aharonian \& H. Voelk eds., AIP, in press (astro-ph/0101382) 
Spada, M., Ghisellini, G., Lazzati, D., \& Celotti, A. 2001, MNRAS, 325, 1559

Tavecchio, F., Maraschi, L., \& Ghisellini, G. 1998, ApJ, 509, 608

Tavecchio, F. et al. 2000, ApJ, 543, 535

Tavecchio, F. et al. 2001, ApJ, 554, 725

Unwin, S. C., Cohen, M. H., Hodges, M. W., Zensus, J. A., \& Biretta, J. A. 1989, ApJ, 340,117

Villata, M. \& Raiteri, C. M. 1999, A\&A, 347, 30

Wehrle, A. E. et al. 1998, ApJ, 497, 178

Wehrle, A. E., Piner, B. G., Unwin, S. C., Zook, A. C., Xu, W., Marscher, A. P., Teräsranta, H., \& Valtaoja, E. 2001, ApJS, 133, 297

This manuscript was prepared with the AAS IATEX macros v4.0. 


\section{Figure Captions}

Fig. 1.- Residuals of the BeppoSAX data fitted with a power law (upper panel) and a broken power law (lower panel); the absorption is free. Note that the weak excess observed at low energy in the first case is partly accounted for in the second one.

Fig. 2.- SED of 3C 279 obtained with simultaneous observations in 1997 January. Short dashed, long dashed, dot-short dashed and dotted curves show the synchrotron, external Compton, synchrotron self-Compton and disk components, respectively (for details of the model see the text). Continuous line is a sum of all the contributions. Points from $10^{12} \mathrm{~Hz}$ to $6 \cdot 10^{13} \mathrm{~Hz}$ are ISO data and come from Haas et al. 1998; the curve don't fit these data because they are not produced in the region to which we refer in this work (as touched on in the text, they may be emitted by dust external to the jet).

Fig. 3.- Overall SEDs of 3C 279 dating back to 1991 June (upper panel) and 1992 December - 1993 January (lower panel). Short dashed, long dashed, dot-short dashed and dotted curves show the synchrotron, external Compton, synchrotron self-Compton and disk components, respectively (for details of the model see the text). Continuous line is a sum of all the contributions. Simultaneous data are from Hartman et al. 1996 (1991), Bonnell et al. 1994 (1991; UV), Maraschi et al. 1994 (1993).

Fig. 4.- Overall SEDs of 3C 279 dating back to 1996 January (pre-flare, upper panel) and 1996 February (flare, lower panel). Short dashed, long dashed, dot-short dashed and dotted curves show the synchrotron, external Compton, synchrotron self-Compton and disk components, respectively (for details of the model see the text). Continuous line is a sum of all the contributions. Simultaneous data are from Wehrle et al. 1998.

Fig. 5.- Comparison between the SEDs of 3C 279 in different levels: the lines reported are 
sums of all the components used to describe the emission observed (synchrotron radiation, SSC, EC and disk emission).

Fig. 6. - $\gamma$-ray vs X-ray flux for different source states. To the former we used fluxes at $167 \mathrm{MeV}$, while the latter has been measured at $3 \mathrm{keV}$. For several states the we have only a maximum and a minimum flux in the X-ray band. Data are from H01 (see this article for the references); we thought right to use as errors in both the ranges the maximum between the values provided and the $5 \%$ of the flux, in order to take into accounts intercalibrations problems. The fit with a straight line provides a slope $1.49 \pm 0.13$, with $\chi_{r}^{2}=2.76$. 
Table 1: BeppoSAX data observation log.

\begin{tabular}{|c|c|c|c|c|c|c|c|c|}
\hline \multirow[b]{2}{*}{ Date } & \multirow[b]{2}{*}{ Start } & \multirow[b]{2}{*}{ End } & \multicolumn{2}{|c|}{$\operatorname{LECS}^{(a)}$} & \multicolumn{2}{|c|}{$\operatorname{MECS}^{(b)}$} & \multicolumn{2}{|c|}{$\operatorname{PDS}^{(c)}$} \\
\hline & & & $\begin{array}{l}\text { Exp. } \\
\left(10^{4} \mathrm{~s}\right)\end{array}$ & $\begin{array}{c}\text { net cts/s } \\
\left(10^{-1}\right)\end{array}$ & $\begin{array}{l}\text { Exp. } \\
\left(10^{4} \mathrm{~s}\right)\end{array}$ & $\begin{array}{c}\text { net cts/s } \\
\quad\left(10^{-1}\right)\end{array}$ & $\begin{array}{c}\text { Exp. } \\
\left(10^{4} \mathrm{~s}\right)\end{array}$ & $\begin{array}{c}\text { net cts/s } \\
\qquad\left(10^{-1}\right)\end{array}$ \\
\hline $13-23 / 01 / 97$ & $22: 55: 25$ & $23: 13: 06$ & 3.116 & $0.67 \pm 0.02$ & 8.473 & $1.14 \pm 0.01$ & 3.653 & $1.11 \pm 0.46$ \\
\hline
\end{tabular}
(a) $0.1-4.5 \mathrm{keV}$
(b) $1.8-10.5 \mathrm{keV}, 3$ MECS units
(c) $15-180 \mathrm{keV}$ 
Table 2: Fits to BeppoSAX Data (LECS+MECS+PDS): first line simple power-law, second line broken power-law, third line power-law+black body model. Errors are quoted at the $90 \%$ confidence level for 1 parameter of interest $\left(\Delta \chi^{2}=2.71\right)$.

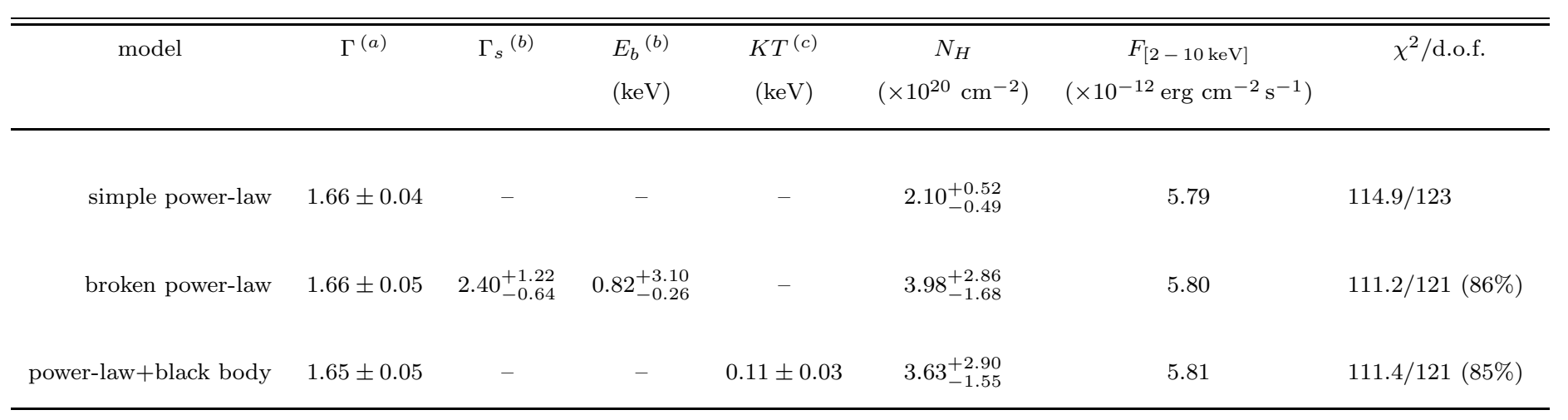

\footnotetext{
(a) photon index, related to the spectral index $\alpha$ (where $F \propto \nu^{-\alpha}$ ) by $\alpha=\Gamma-1$.

(b) break energy and high energy photon index for the broken power-law model.

(c) temperature for the black body model.
} 
Table 3: Optical observation log.

\begin{tabular}{|c|c|c|c|}
\hline \multirow[t]{3}{*}{ Date } & \multicolumn{3}{|c|}{ Magnitude } \\
\hline & $\mathrm{R}$ & $\mathrm{V}$ & $\mathrm{B}$ \\
\hline & $\left(4.36 \cdot 10^{14} \mathrm{~Hz}\right)$ & $\left(5.37 \cdot 10^{14} \mathrm{~Hz}\right)$ & $\left(6.76 \cdot 10^{14} \mathrm{~Hz}\right)$ \\
\hline $11 / 01 / 97$ & $15.50 \pm 0.03$ & $15.93 \pm 0.04$ & - \\
\hline $12 / 01 / 97$ & $15.46 \pm 0.02$ & $15.88 \pm 0.03$ & $16.31 \pm 0.07$ \\
\hline $13 / 01 / 97$ & $15.37 \pm 0.03$ & $15.84 \pm 0.04$ & $16.37 \pm 0.05$ \\
\hline $14 / 01 / 97$ & $15.50 \pm 0.02$ & - & - \\
\hline $15 / 01 / 97$ & $15.48 \pm 0.04$ & $15.99 \pm 0.05$ & - \\
\hline $16 / 01 / 97$ & $15.48 \pm 0.02$ & $15.95 \pm 0.07$ & - \\
\hline $17 / 01 / 97$ & $15.49 \pm 0.02$ & $15.98 \pm 0.03$ & - \\
\hline \multirow[t]{4}{*}{$18 / 01 / 97$} & $15.52 \pm 0.02$ & $16.01 \pm 0.04$ & $16.55 \pm 0.05$ \\
\hline & $15.57 \pm 0.02$ & $15.98 \pm 0.03$ & - \\
\hline & $15.50 \pm 0.03$ & $16.07 \pm 0.04$ & - \\
\hline & $15.50 \pm 0.04$ & - & - \\
\hline
\end{tabular}


Table 4: Parameters used for the emission model described in the text; the BLR luminosity is $L_{b l r}=6 \cdot 10^{44} \mathrm{erg} \mathrm{s}^{-1}$. We assume a blob with $R=5 \cdot 10^{16} \mathrm{~cm}$ in this radiation field diluted in a region with radius $R_{b l r}=4 \cdot 10^{18} \mathrm{~cm}$. The electron distribution extended from $\gamma_{\min }=1$ to $\gamma_{\max }=5 \cdot 10^{4}$, and the angle of view is $\theta=3^{\circ}$. These values was kept fixed in all fits (see the text for more details).

\begin{tabular}{|c|c|c|c|c|c|c|}
\hline$\Gamma$ & $\delta^{(a)}$ & $\begin{array}{c}B \\
(G)\end{array}$ & $\begin{array}{c}\gamma_{b} \\
\left(\times 10^{2}\right)\end{array}$ & $n_{1}$ & $n_{2}$ & $\begin{array}{c}k \\
\left(\times 10^{3} \mathrm{~cm}^{-3}\right)\end{array}$ \\
\hline
\end{tabular}

1991

\begin{tabular}{ccccccc}
\hline 13 & 17.8 & 0.6 & 5.5 & 1.6 & 4.7 & 5 \\
& \multicolumn{7}{c}{$\mathbf{1 9 9 3}$} \\
\hline 6 & 10.9 & 0.7 & 4.5 & 1.6 & 4.4 & 5.6 \\
& \multicolumn{7}{c}{$\mathbf{1 9 9 6}$ pre - flare } \\
\hline 11 & 16.5 & 0.5 & 5 & 1.6 & 4.7 & 5
\end{tabular}

1996 flare

\begin{tabular}{lllllll}
\hline 17 & 19 & 0.5 & 4.9 & 1.6 & 4.7 & 5.3 \\
& & & & & & \\
& & & & & & \\
\hline 7 & 12.3 & 0.5 & 6.0 & 1.6 & 4.2 & 4.5 \\
\hline
\end{tabular}

(a) completely determined if $\Gamma$ and $\theta$ are fixed; therefore this is not a free parameter 

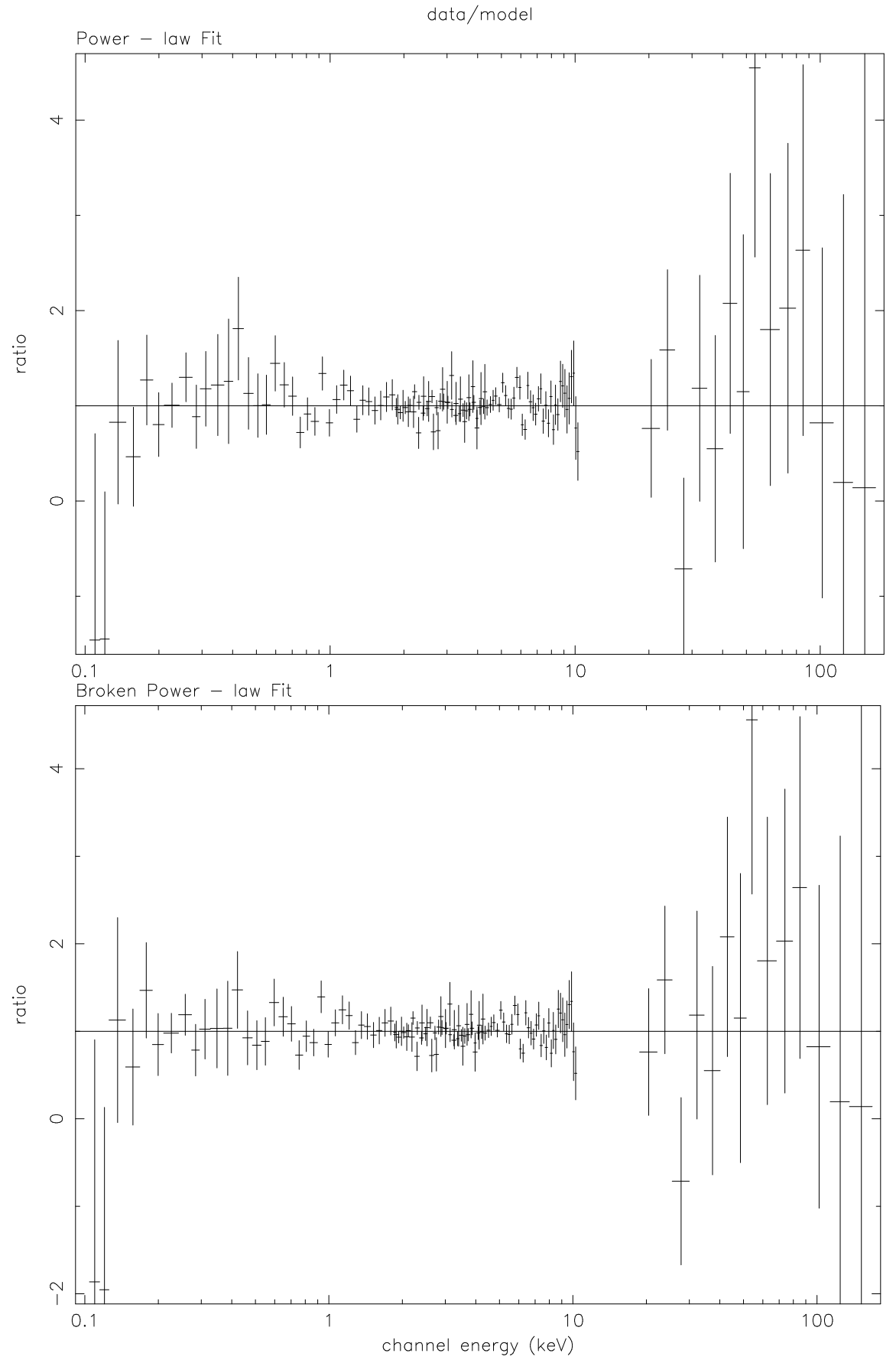


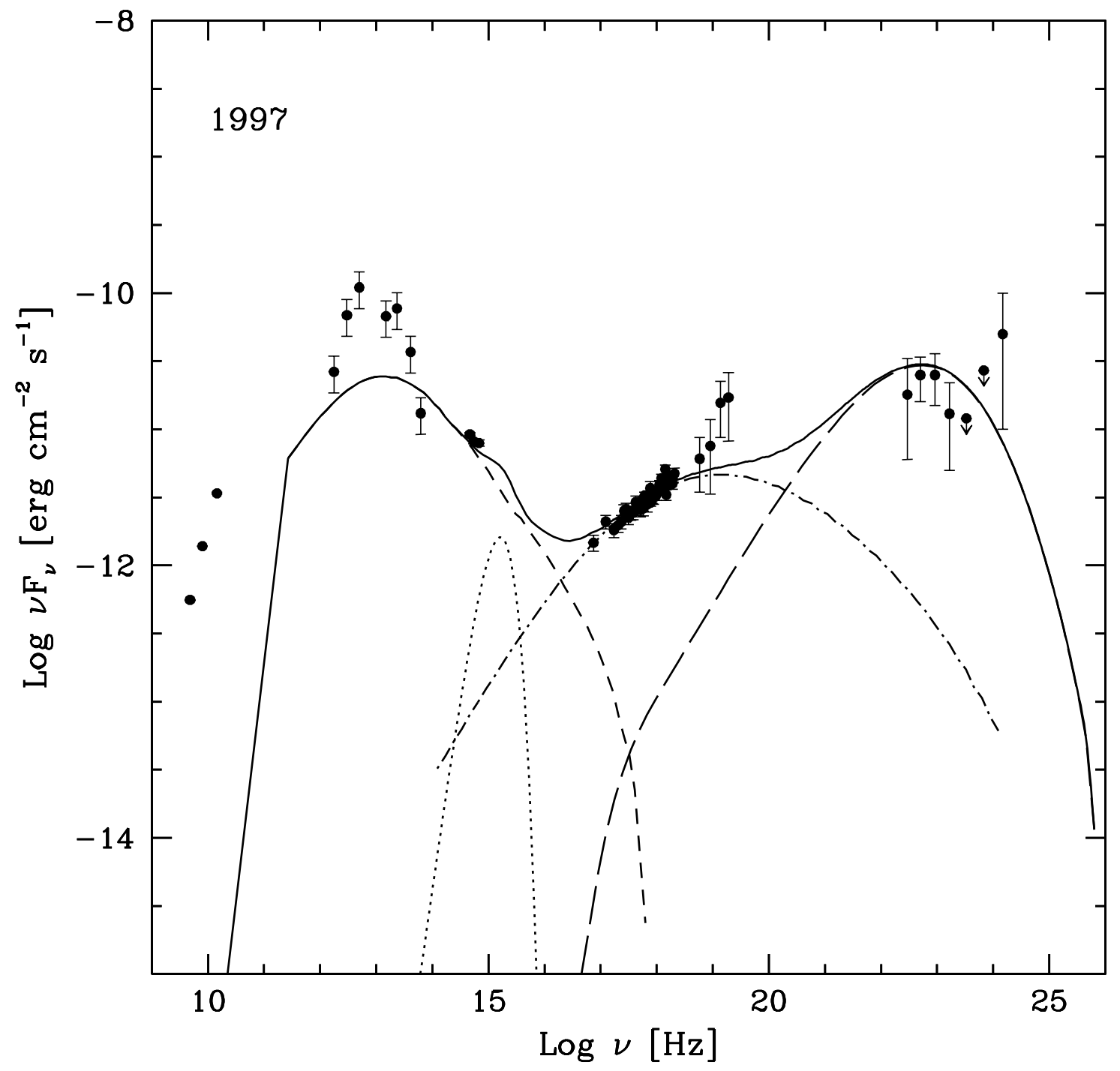



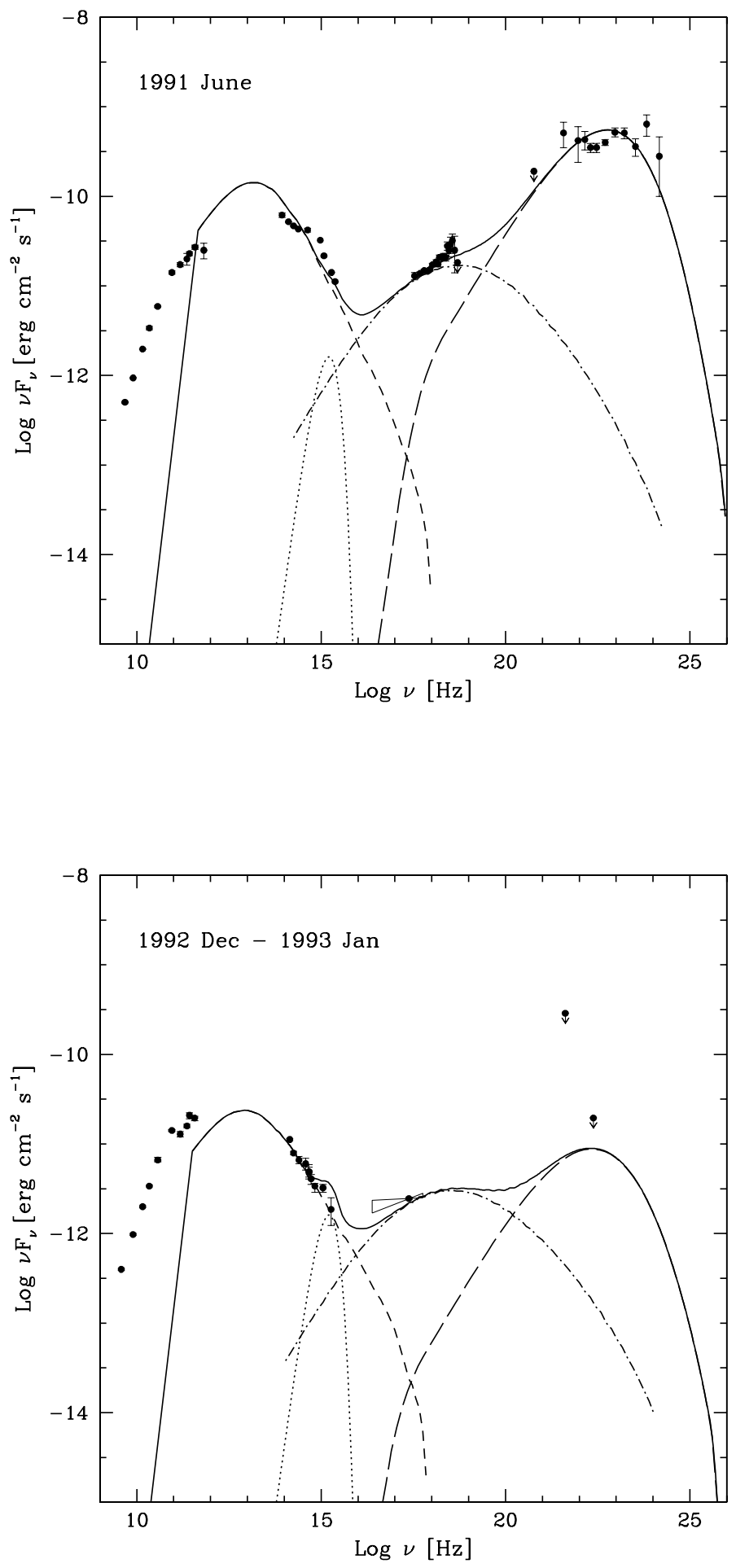

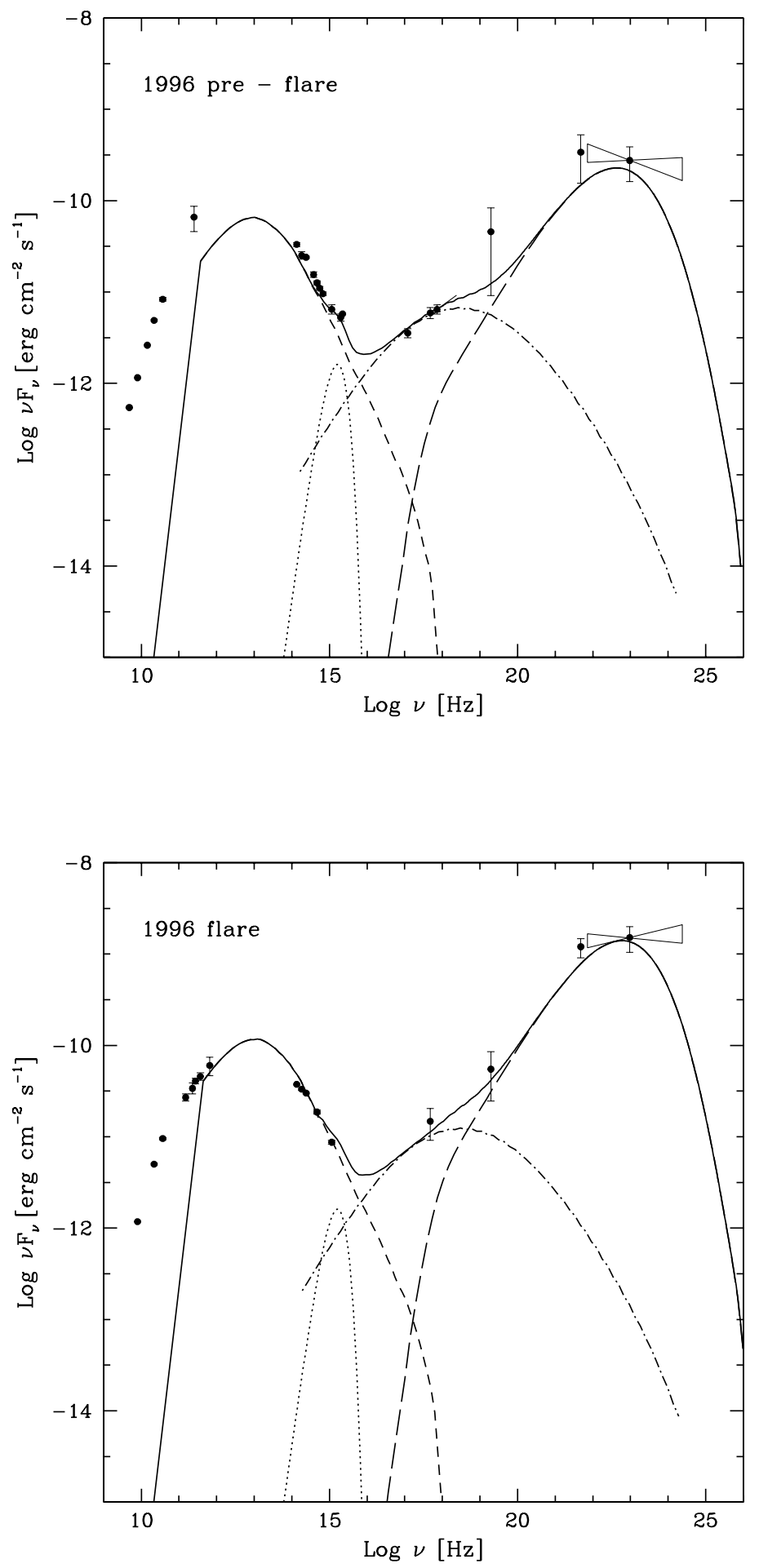


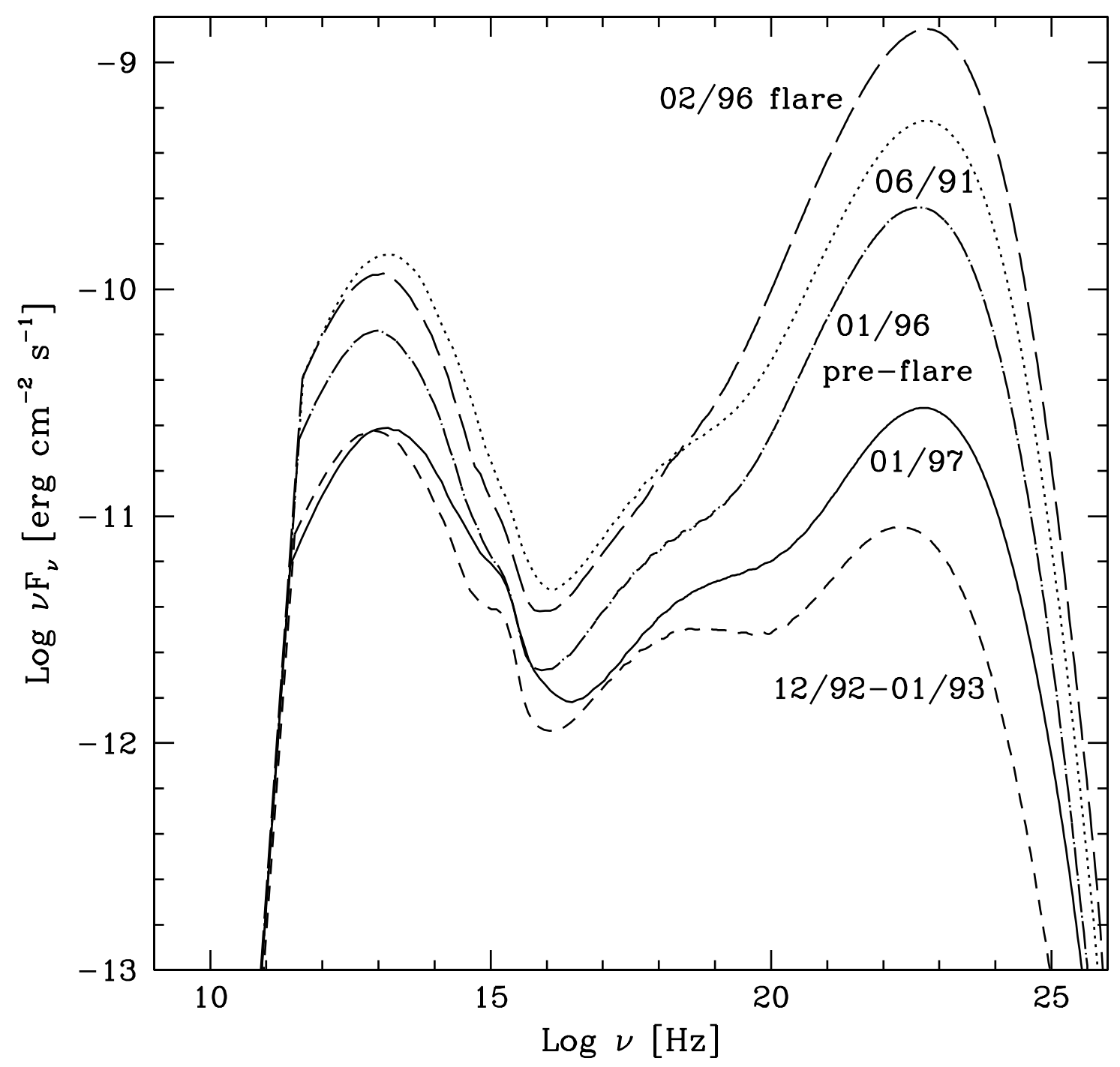




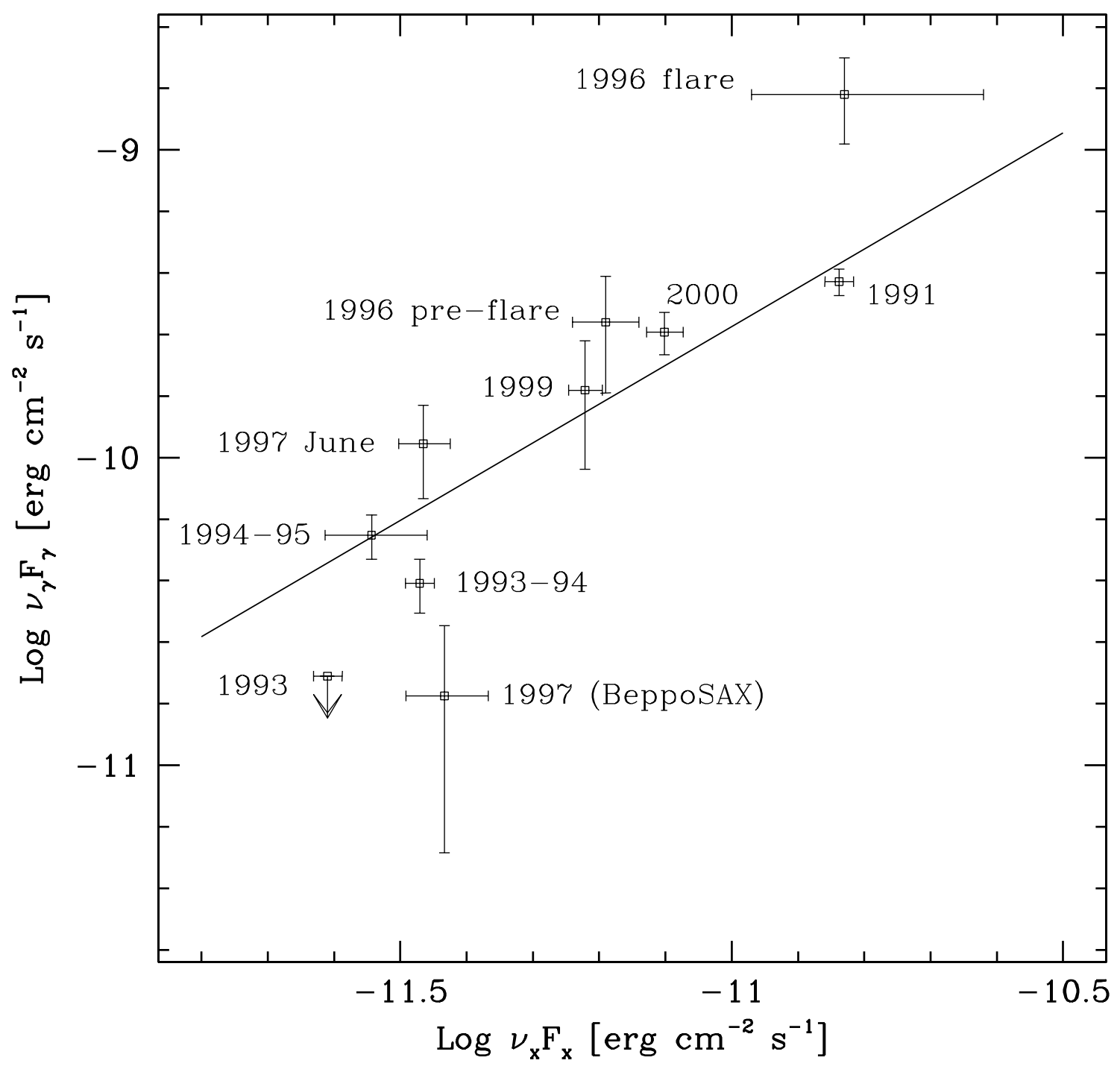

